\title{
Multiplexing in 5G Mobile Technology
}

\author{
Eng. Khawlah Juma’a Rashid Al-Hamdan, \\ Transmission Department, \\ Higher Institute of Telecommunication and Navigation, \\ Kuwait
}

\begin{abstract}
Multiplexing of 5G mobile technology is an essential aspect of technological advancement that has made the communication to reach high levels. It applies two main categories of connectivity that include Partial and full connectivity. This paper proposes a hybrid structure for hybrid beamforming in millimeter-wave ( $\mathrm{mm}$ Wave) large systems. In this structure, the antenna arrays at the transmitter and the receiver comprise of multiple sub-arrays. Each of the sub-arrays connects to several radio frequency (RF) chains. Also, each RF chain is linked with all the antennas matching to the sub-array. In this structure, the precoding matrix optimization constraint is decomposed into numerous precoding sub-matrix optimization constraints through successive cancelation. Then, analog precoders and near-optimal hybrid digital are planned by factoring the precoding sub-matrix for individual sub-arrays. Additionally, the performance of the proposed hybrid connected structure is compared with the current partially and fully connected structures based on the required number of base shifters and spectral efficiency. Finally, the results generated from the simulation are presented to indicate the difference between the spectral efficiency of the partially connected hybrid structure and the fully connected one. Moreover, the algorithm for the hybrid combined structure can achieve higher energy proficiency than present algorithms for the full and partly linked structures.
\end{abstract}

\section{INTRODUCTION}

The advent of $5 \mathrm{G}$ has been dubbed as one of the most efficient and reliable due to its architectural agility. The $5 \mathrm{G}$ network architecture has several modifications which not only make it simpler to use but also it increases the system efficiency by reducing the over-reliance between the access and other fundamental networks and the hardware and software elements of the network functions. For instance, the presence of Control Plane User Separation (CUPS)in the 5G network provides an enhanced architecture which helps in separating the user's signaling and other data traffic in the system. Further, 5G has the capability of supporting the Ultra Reliable Low Latency Communication (URLLC) devices, mainly employed in telesurgery applications and even in the industrial automation [1]. Another important feature which sets apart the $5 \mathrm{G}$ network from other networks is that $5 \mathrm{G}$ comes with a Network Slicing feature which makes it possible for network infrastructure to be partitioned into different virtual systems. Last in this list is the "Multi-Connectivity" capability which helps different types of networks such as, $5 \mathrm{G}, \mathrm{WiFi}$, LTE to be accessed from a single interface, e.g., from radio to the core.

The concept of "Multi-Connectivity" defines the purpose of this article which generally aims at reporting on Multiplexing feature in 5G mobile technology. Specifically, the story has two primary objectives; to discuss the concept of waveform multiplexing in $5 \mathrm{G}$ and to analyze and appraise the Multiplexing millimeter-wave beams for $5 \mathrm{G}$ technology.
The article will start by defining the idea of Multiplexing, then discuss extensively on waveform multiplexing for $5 \mathrm{G}$ and lastly analyze the concept of Multiplexing millimeter-wave beams for $5 \mathrm{G}$ technology [2]. Finally, the final section of this article will conclude the entire story

\section{MULTIPLEXING}

Multiplexing or MUXing as it is known in telecommunication and computer networks refer to a process through which several or multiple message signals (either analog or digital data streams) are transmitted as one signal in a single carrier. Usually, signals are transmitted simultaneously by converting them into common signals of similar bandwidth. Simply, Multiplexing can also be defined as the "ability to transfer data coming from several pairs of equipment (transmitters and receivers) called low-speed channels on a single physical medium (called the high-speed channel). One of the main advantages of $5 \mathrm{G}$ network and as illustrated earlier is that unlike other network generations;5G provides an enhanced architecture which helps in separating the user's signaling and other data traffic in the system and this zero down to multiplexing as multiple users use one bandwidth of same communication medium [3].Different techniques of multiplexing exist, and their exact configuration is dependent on the mode of the data transmission. As such, different types of multiplexing technologies are available primarily for the $5 \mathrm{G}$ network, but their exact configuration is what makes them vary.

\section{I.1 Waveform multiplexing for $5 G$}

The concept of waveform multiplexing has been subjecting a great debate by various scholars especially when evaluating and measuring the functionality of the $5 \mathrm{G}$ network [4]. For this article, waveform multiplexing will be presented as a "technique system of the multiplexing of numerologies operating efficiently." Waveform multiplexing is a type of sub-band system which depicts the following attributes:

I.1.1 Multiplexing of Waveforms on Sub-bands with Scalable Subcarrier Spacing; in this, a single system supports different applications such as URLLC, eMBB, and the massive Machine-type Communication (mMTC) such that all these applications transmit different data on one frequency band [5]. Every sub-band is comprised of various waveforms which have different subcarrier spacing and therefore each sub-band can use different waveforms sets available.

I.1.2 Dynamic CP: one of the most significant advantages of $5 \mathrm{G}$ waveforms is that they have a low OOBE. This phenomenon is crucial for the suggested waveform multiplexing as it can be able to utilize various $\mathrm{CP}$ lengths which were not possible with the OFDM system which mainly used a fixed CP length to maintain its orthogonality 
[6]. The dynamic $\mathrm{CP}$ is of great optimizer especially in the $5 \mathrm{G}$ communication as it helps the band to vary when the cell sizes shirk and the delay spreads.

I.1.3 Minimum Guard Band between Sub-bands; the low OOBE helps to minimize the guard band between the subbands, and helps to raise the spectrum usage especially for $5 \mathrm{G}$, unlike the OFDM. For instance, the 3GPP commission which is evaluating the $5 \mathrm{G}$ system has been considering the zero-guard band for the

From the three characteristics discussed above, it is clear that the OFDM waveform although well-known, studied and applied widely in 4G (LTE). However, the OFDM has several areas which actively bring inter-carrier interference, and this inhibits its application in the 5G networks [7]. An alternative to OFDM, Filter-bank Based Multi-carrier (FBMC) has been proposed as its side-lobes are usually weaker and this helps to reduce the Inter-carrier interference, and therefore any system using FBMC waveform is more suited for $5 \mathrm{G}$ in this respect.

\section{FBMC}

Two types FBMC- filtered multi-tone (FMT) and the staggered multi-tone (SMT) exists but both functions on the fundamental principle described above. However, SMT has the capability of achieving a high spectral efficiency, and therefore it is more preferred than the FMT. For instance, for SMT to acquire a time-frequency competence of 1 , it must stagger the multi-carrier symbols timely, and it must apply the offset-QAM. When there are complex symbols which must be conveyed such as (QPSK, xQAM), the transmitter breaks the symbols into imaginary and real parts and then modulates these parts on consecutive symbols [8]. Usually, the SMT does not apply the orthogonal, especially with the complex plains. Although different sub-carriers in the system introduces some interference to those in their neighborhood, the intervention is usually minimized to zero digits by the application of offset-QAM which helps to discard any received symbol which does not have any data.

FBMC relies heavily on the "filtering functionality on a per sub-carrier basis." Therefore, the frequency response of the filter applied in the system must be tight. As such, the system requires relatively long filter lengths which are either three or four times the length of the symbol itself are employed. As a result, the system must have a long ramp up and ramp down areas to facilitate the transmission of bursty data in case the conveyance of the same if required. Consequently, the need for great filters and the introduction of the interference by the neighboring subcarriers are the main drawbacks of FBMC systems [9]. Although, there are more systems and approaches which are more advanced, the application of FBMC in the $5 \mathrm{G}$ network can prove to be advantageous as other methods might be expensive than others.

\section{UNIVERSAL-FILTERED MULTI-CARRIER (UMFC)}

The robustness depicted by the UMFC especially in overcoming the inter-carrier interference discussed above has made the technique to be applauded as it matches perfectly with the $5 \mathrm{G}$ network requirements. UFMC can be defined as a "multi-carrier signal format with orthogonal subcarriers to handle the problem of loss of orthogonality at the receiver end." Practically, UMFC can be said to be a generalization of filtered OFDM and FBMC techniques combined. However, the UMFC system applies sub-band filters which help the system to accommodate short burst communication, which is comparable to FBMCas it functions using the same principle. As a result of the application of sub-bands filters in the UFMC, the system has a much higher spectral efficiency as compared to other techniques discussed earlier. Moreover, the system does not rely on cyclic prefix insertion, and therefore there is no repetition of bits which are similar and therefore, the system uses the entire available spectrum efficiently. Another important feature which helps UFCM to be more compatible with the $5 \mathrm{G}$ network is that the technique has fewer side lobes and this helps the entire system to have less interference on the neighboring sub-carriers. Usually, OFDM conveys data by using large blocks of independently modulated sub-carriers and these increases the Peak-toAverage Power Ratio (PAPR) especially when they are introduced in phase. However, the total bandwidth in UFMC is divided into sub-bands and this helps to decrease the PAPR as subcarriers adding accumulation in the system is less as compared in OFDM for instance. As such, UFMC has a low PAPR than OFDM which makes the earlier method to be promoted for the $5 \mathrm{G}$ network.

\section{MULTIPLEXING MILLIMETER-WAVE BEAMS FOR 5G TECHNOLOGY}

The $5 \mathrm{G}$ technology is scheduled to be launched officially by 2020, and therefore scientists are working round the clock to identify an innovative solution which will help in enabling the optimum 5G era. Millimeter wave(mmWave)which have been used in communication especially for radio has received lion share attention as a potential for $5 \mathrm{G}$ network as the system can be able to transmit bigger data at gigabit-persecond. The mmWave band can be defined as a frequency range which starts at $30 \mathrm{GHz}$ to $300 \mathrm{GHz}$ and sometimes the system is also known as the extremely high frequency (EHF). Further, EHF is also the highest electromagnetic radiation radio frequency band. The $3-30 \mathrm{GHz}$ spectrum is known as super high frequency (SHF), and they have similar characteristics with the EHF which operates at the 3-300GHz spectrum, and they are known as mmWaves bands. Moreover, these bands share similar wavelengths ranging from 1$100 \mathrm{~mm}$. The presence of more significant spectrum at the mmWave frequencies such as local multipoint distribution service $(28-30 \mathrm{GHz})$, the license-free band $(60 \mathrm{GHz})$, and the E-band (71-76 GHz) among others are available for the $5 \mathrm{G}$ utilization. Further, mmWaves can be reused for short distance communications, and this is aided by the high attenuation in the free space penetration [10]. However, the mmWave band has several vital concerns which affect the transmission of data using this method as it is prone to lose data under several conditions such as; atmospheric gaseous losses, rain, and the attenuation brought by the free space propagation.

\section{APPLICATIONS OF MMWAVE COMMUNICATIONS}

\section{V.1 Small Cell Access}


In order to satisfy the oblivious expanding mobile traffic demand, huge densification of small cells has been suggested to raise the network capacity in the future. The small cells which have been released under-laying the macro-cells (WLANs or WPANs) provide brilliant capabilities for the $5 \mathrm{G}$ network prospects. One of the main advantages of mmWave small cells is that they can offer multi-gigabit rates. Moreover, mmWavesmall cells have wideband which can be harnessed for multimedia applications such as HDTV, highspeed data transfer, and wireless gaming among others. For instance, Ghoshet al. in their study illustrated a case in which they deployed mmWave bands such as 28 up to $8-86 \mathrm{GHz}$ for a $5 \mathrm{G}$ enhanced local area (eLA) access, and they observed that the eLA system achieved more than 10Gbps peak data rates while its edge data was in excess of 100Mbps [11]. From this eLA simulation, it is clear that mmWave can be used in the $5 \mathrm{G}$ communication.

\section{V.2 Cellular Access}

Studies have shown that a large bandwidth in mm Wave can have a higher coverage capacity provided there is the presence of infrastructure deployment is dense. The deviceto-device (D2D) has been seen to be useful as it helps to save power and also reinforce the spectral efficiency, a property which can help in unleashing the 5G network readily [12]. Since the cellular access mainly relies on $28 \mathrm{GHz}$ and $38 \mathrm{GHz}$ supported by cell sizes which are of the order $200 \mathrm{~m}$, its effectiveness can be improved by pointing the directional antennas in the strongest transmit and receive directions.

\section{V.3 Wireless Backhaul}

One of the main drawbacks of using small cells which are densely deployed for $5 \mathrm{G}$ is that it makes it nearly impossible to interlink or connect two $5 \mathrm{G}$ base stations or and to the network by using the fiber-based backhaul due to higher costs involved. However, a higher speed wireless backhauling mmWave bands such as $81-86 \mathrm{GHz}$ have been proved to be more cost-effective and user-friendly [13]. For instance, am E-band backhaul has been established to offer the best solution as it can be able to provide higher speeds between two or more base stations and even between the small cell base stations. Another study by Taori et al. suggests that "inband wireless backhaul solution, where the backhaul and the access are multiplexed on the same frequency band." In this system, they concluded that the "joint design of the access and backhaul networks would optimize the resource allocation further." Although this system has proven as a possible solution for the 5G network, other band possibilities should be investigated to get an optimum solution.

\section{V.4 MIMO at mmWave Frequencies}

MIMO technique has also received huge interest in mmWave communications as it has been observed to offer a trade-off in multiplexing and the diversity gains. To have higher yields of MIMO, a hybrid beam-forming structure have been devised to help in overcoming the propagation loss in mmWave bands.

Hybrid Beam-forming Structure

Baseband beamforming architecture provides a massive antenna guide that enables multi-user connections, multi- stream with a wide range of transmission modes [14]. However, it becomes a significant challenge to the designers, especially when more than 100 antennas are involved. Over 100 power-hungry converters are required to operate the various antennas that convert digital signals to analog signals and vice versa. The high-power requirement increases power consumption and hardware complexity that makes the architecture to be impractical for the designs. Hence, digital beamforming architecture is used in mobile applications in downlink transmitters. It is worth noting that digital beam forming high power consumption makes the system to be unique in that some of its receivers enhance the mitigation of uplink interference between the cells. RF beamforming is structured in such a way that all the precoding is done in the side of RF. It is more advantageous than digital beamforming because it uses lower power and has a lower hardware complexity. Additionally, the phase shifters in CMOs are characterized by high performance, low level of amplitude about frequency and high phase variation about voltage control. Some of the ancient $5 \mathrm{G}$ prototyping systems under the frequency band of $63.5 \mathrm{GHz}$ has been developed using RF beamforming design.

\section{CONCLUSION}

The wake of $5 \mathrm{G}$ advancement has been identified as reliable and effective. The architecture of the network has various adjustments which make it easy to use and increases the efficiency of the system by reducing dependence between the access and the other basic fundamental hardware and software elements and the networks. Multiplexing which is a process in which multiple message signals as one single signal in a single carry. The signals are transmitted simultaneously by converting them into a common signal of similar bandwidth. Indeed, there are different techniques of multiplexing for the $5 \mathrm{G}$ network, but their configuration makes them contrast. In the case of waveform multiplexing for the $5 \mathrm{G}$ is a type of sub-band system which shows characteristics such as Dynamic Cp and Minimum Guard Band between Sub-bands. The attributes have numerous advantages such as a low OOBE which helps to minimize the guard band between the sub-bands in Minimum Guard Band between Sub-bands. Moreover, the application of the multiplexing millimeter-wavelength in communication the small cell access densification has been suggested to satisfy the expanding traffic demand. Various countries should work together to find the suitable ways to encourage technological development as it is leading to increased efficiency in numerous sectors

\section{REFERENCES}

[1] Blanco, B., Fajardo, J. O., Giannoulakis, I., Kafetzakis, E., Peng, S. Pérez-Romero, J., ... \& Sfakianakis, E. (2017). Technology pillars in the architecture of future 5G mobile networks: NFV, MEC, and SDN. Computer Standards \& Interfaces, 54, 216-228.

[2] Chen, S., \& Zhao, J. (2014). The requirements, challenges, and technologies for $5 \mathrm{G}$ of terrestrial mobile telecommunication. IEEE communications magazine, 52(5), 36-43.

[3] Dai, L., Wang, B., Yuan, Y., Han, S., Chih-Lin, I., \& Wang, Z. (2015). Non-orthogonal multiple access for 5G: solutions, challenges, opportunities, and future research trends. IEEE Communications Magazine, 53(9), 74-81. 
[4] Hossain, E., \& Hasan, M. (2015). 5G cellular: key enabling technologies and research challenges. IEEE Instrumentation \& Measurement Magazine, 18(3), 11-21.

[5] Liang, C., Yu, F. R., \& Zhang, X. (2015). Information-centric network function virtualization over $5 \mathrm{G}$ mobile wireless networks. IEEE Network, 29(3), 68-74.

[6] Mitra, R. N., \& Agrawal, D. P. (2015). 5G mobile technology: A survey. ICT Express, 1(3), 132-137.

[7] Osseiran, A., Monserrat, J. F., \& Marsch, P. (Eds.). (2016). 5G mobile and wireless communications technology. Cambridge University Press.

[8] Roh, W., Seol, J. Y., Park, J., Lee, B., Lee, J., Kim, Y., ... \& Aryanfar, F. (2014). Millimeter-wave beamforming as an enabling technology for $5 \mathrm{G}$ cellular communications: Theoretical feasibility and prototype results. IEEE communications magazine, 52(2), 106113.

[9] Xiang, W., Zheng, K., \& Shen, X. S. (Eds.). (2016). 5G mobile communications. Springer.

[10] Zhang, X., Cheng, W., \& Zhang, H. (2015). Full-duplex transmission in PHY and MAC layers for 5G mobile wireless networks. IEEE Wireless Communications, 22(5), 112-121.

[11] Agiwal, M., Roy, A., \& Saxena, N. (2016). Next generation 5G wireless networks: A comprehensive survey. IEEE Communications Surveys \& Tutorials, 18(3), 1617-1655.

[12] Taori, R., \& Sridharan, A. (2015). Point-to-multipoint in-band mmwave backhaul for 5G networks. IEEE Communications Magazine, 53(1), 195-201.

[13] Shariatmadari, H., Ratasuk, R., Iraji, S., Laya, A., Taleb, T., Jäntti, R., \& Ghosh, A. (2015). Machine-type communications: current status and future perspectives toward $5 \mathrm{G}$ systems. IEEE Communications Magazine, 53(9), 10-17.

[14] Hong, S., Brand, J., Choi, J., Jain, M., Mehlman, J., Katti, S., \& Levis, P. (2014). Applications of self-interference cancellation in 5G and beyond. IEEE Communications Magazine, 52(2), 114-121.

[15] Geetha, B. T., Vidhya, B., Pushpalatha, K., \& Priyadharshini, M. (2017, September). A comparative study of 5G technology with other generations of mobile technologies. In 2017 IEEE International Conference on Power, Control, Signals and Instrumentation Engineering (ICPCSI) (pp. 1778-1782). IEEE. 